\title{
BMJ Open Health literacy in people with venous leg ulcers: a protocol for scoping review
}

\author{
CD Weller (D) , ${ }^{1}$ Victoria Team (D) , ${ }^{1}$ Sebastian Probst (D) , ${ }^{2}$ Georgina Gethin (i) , ${ }^{3}$ \\ Catelyn Richards, ${ }^{1}$ Jane Sixsmith (D) , ${ }^{4}$ Louise Turnour, ${ }^{1}$ Ayoub Bouguettaya (i) ${ }^{5}$
}

To cite: Weller CD, Team V, Probst S, et al. Health literacy in people with venous leg ulcers: a protocol for scoping review. BMJ Open 2021;11:e044604. doi:10.1136/ bmjopen-2020-044604

- Prepublication history and additional supplemental material for this paper are available online. To view these files, please visit the journal online (http://dx.doi.org/10.1136/ bmjopen-2020-044604).

Received 10 September 2020 Revised 08 February 2021 Accepted 12 March 2021
Check for updates

(c) Author(s) (or their employer(s)) 2021. Re-use permitted under CC BY-NC. No commercial re-use. See rights and permissions. Published by BMJ.

\section{${ }^{1}$ School of Nursing and}

Midwifery, Monash University, Melbourne, Victoria, Australia

${ }^{2} \mathrm{HES}$-SO Genève, Genève,

Switzerland

${ }^{3}$ School of Nursing and

Midwifery, National University of Ireland Galway, Galway, Ireland

${ }^{4}$ Health Sciences, National

University of Ireland Galway,

Galway, Ireland

${ }^{5}$ School of Psychology, University of Birmingham Edgbaston Campus, Birmingham, UK

Correspondence to

Dr CD Weller;

carolina.weller@monash.edu

\section{ABSTRACT}

Introduction Chronic venous leg ulcer (VLU) healing is a complex clinical problem. It requires intervention from skilled, costly, multidisciplinary wound-care teams, working with patients to manage their care. Compression therapy has been shown to help heal venous ulcers and to reduce recurrence, with some evidence suggesting the value of exercise as well. These activities require health education and health literacy $(\mathrm{HL})$ as patients must process, understand and consistently apply health information for successful self-management. Research suggests that those most vulnerable to VLUs also tend to have limited $\mathrm{HL}$, but there have been no reviews examining the state of $\mathrm{HL}$ in patients with previous or active VLUs. This scoping review aims to examine the level of $\mathrm{HL}$ in VLU patients and how HL may link to self-management behaviours (particularly exercise and compression adherence), and their VLU healing generally.

Methods and analysis We will use Preferred Reporting Items for Systematic Reviews and Meta-Analyses Scoping Review guidelines and the Levac methodology framework to explore eligible papers that examine the effect of $\mathrm{HL}$ on their exercise and compression adherence. Electronic databases will be searched (MEDLINE, EMBASE, the Cochrane Library, PsycInfo and Health, OpenGray), examining for all papers on these subjects published between 2000 and 2020. All studies describing compression and or exercise during VLU management will be included. Study characteristics will be recorded; qualitative data will be extracted and evaluated. Quantitative data will be extracted and summarised.

Ethics and dissemination We will disseminate results through peer-reviewed publications. We will use data (ie, journal articles) from publicly available platforms; so, this study does not require ethical review. The consultation step will be carried out with patients, carers and health professionals as part of an established wound consumer group.

\section{INTRODUCTION}

Venous leg ulcers (VLUs) are chronic skin ulcers mainly affecting the gaiter area, most often caused by continuous venous hypertension or chronic venous insufficiency. ${ }^{1}$ VLUs are a common health condition, affecting approximately $1 \%-3 \%$ of people globally. ${ }^{2-4}$ This is often due to persistent high blood pressure in varicose veins. ${ }^{56}$ The prevalence of VLU increases with age, doubling among
Strengths and limitations of this study

This scoping review (ScR) protocol is the first to focus on the role of consumers' health literacy in venous leg ulcer management.

- In order to ensure a systematic approach to searching, screening and reporting, we use the Preferred Reporting Items for Systematic Reviews and MetaAnalyses extension for ScRs tool, the most current guidance on conducting ScRs.

- We have included a comprehensive search strategy and data extraction template.

- The studies included in the review will be appraised for quality.

- Studies published outside of the indicated databases may be missed.

those aged over 65 years. ${ }^{7}$ People with VLU often have various other comorbidities, including arterial hypertension, obesity, non-insulin-dependent diabetes and dyslipidaemia. ${ }^{8}$ VLU healing, defined as complete wound re-epithelisation, ${ }^{9}$ is often slow. The chance of recurrence of a healed VLU is high. ${ }^{10}{ }^{11}$ The cycle of healing and frequent recurrent episodes has significant economic impact ${ }^{412-14}$ and severely affects VLU patients' health and well-being, including that of their families. ${ }^{15-17}$ Despite advances in treatments, sustained healing of VLU continues to be an ongoing problem for patients, health services and health systems. ${ }^{18}$

Current best practice recommendation for people with active VLU is the use of compression therapy unless the patient has arterial insufficiency, where it is contraindicated. ${ }^{1}$ Compression therapy promotes VLU healing by reducing the hydrostatic pressure in lower limbs, enhancing venous return ${ }^{1}$ and preventing venous stasis. Research suggests that the use of below-knee multi-component compression is efficacious and effective. ${ }^{19-21}$ Consistent compression therapy is recommended to prevent VLU recurrence. ${ }^{1}{ }^{21}$ Pharmaceutical, surgical, physiotherapy and other methods can be used as adjunctive to compression, although the evidence of their 
effectiveness is limited. ${ }^{22}$ Other recommendations often include appropriate physical activity, adequate nutrition and leg elevation. ${ }^{1}$ Of these, physical activity has received mixed evidence on its efficacy when combined with compression. ${ }^{2324}$ However, it is still recommended to patients with VLUs to improve VLU healing and reduce the risk of VLU recurrence. ${ }^{24} 25$

The best practice recommendations (exercise, compression) for treatment require significant patient involvement; however, patient adherence to the VLU management recommendations is often suboptimal. ${ }^{23}$ To optimise healing outcomes, VLU patients should follow the management plan and understand the importance of compression and other recommendations. The management plan should be developed in collaboration with patients in a standard consultation ${ }^{126}$ because shared decision making is paramount for a faster healing outcome. ${ }^{27}$ An informed patient can participate as an essential partner in the VLU management process. ${ }^{28}$ The patient's role is complementary to their healthcare professional's role, as they monitor symptoms, adhere to compression and adopt health behaviours, following the advice of health professionals. ${ }^{29}{ }^{30}$ However, the extent to which patients can follow the advice varies, partially based on their HL.

Patient HL is defined as 'the ability to obtain, process and understand basic health information and services needed to make appropriate health decisions and follow instructions for treatment., ${ }^{17} 18$ There is a distinction between general health literacy (HL) often assessed through population-level surveys, ${ }^{31}$ and specific HL which deals with health skill and knowledge specific to the condition or disease. ${ }^{29}$ General HL scales are often used to assess a patient's general capabilities in navigating their health environment (eg, where do you go for medical advice), often for the purpose of directing health policy at a population scale. Meanwhile, specific HL scales assess individual capabilities in dealing with a specific condition, like heart disease or diabetes. Both general and VLUspecific HL may affect VLU outcomes through affecting patient's adoption of health behaviours. Improvements in general and specific HL may improve patient knowledge and understanding of the benefits of adhering to VLU self-management recommendations ${ }^{25-27}$ and support patients to adopt healthy behaviours in line with the agreed plan. For example, when choosing compression hosiery, patients may rely on HL to critique the options based on their analysis of comfort ${ }^{28}$ which may be opposed to achieving maximum therapeutic benefit. ${ }^{30}$ Furthermore, HL may enhance their compression application skills due to improved understanding of the manufacturer's instructions. ${ }^{21}{ }^{32}$ Finally, improved HL may influence patient understanding that lifelong compression hosiery is recommended to prevent VLU recurrence. ${ }^{23}$

\section{Study rationale}

Recent research has indicated that people vulnerable to VLUs, those with VLUs and those with other comorbidities tend to have concurrent deficits in HL. ${ }^{33}$ VLU incidence increases with age ${ }^{13}$ and older people have been shown to have limited general HL. ${ }^{34}$ For example, although the 2015 European health literacy survey showed that respondents received an average score of 33.8/50 (demonstrating 'sufficient' HL), the majority (58\%) of people aged over 66 years had limited HL, compared with less than half of the general population. ${ }^{31}$ A possible reason is internet usage. Though internet use is proportional to increased HL, ${ }^{35}$ current research consistently reports that older adults prefer to learn from their healthcare professionals, ${ }^{36}$ as opposed to independent learning through the use of the internet. ${ }^{37}$ Qualitative research has shown that VLU patients often discuss the volume of information and skills that are needed for self-management on VLU development as a significant burden, as VLU selfmanagement can be complex. ${ }^{38}$ The education needs of VLU patients are not well understood ${ }^{39}$ resulting in unmet HL needs. In general, limited HL in adults is associated with reduced adherence to treatment and health recommendations, poorer health outcomes and increased cost of medical treatment, ${ }^{40}$ especially among older adults. ${ }^{41}$ Furthermore, checking on patient understanding is not a routine practice for healthcare professionals, ${ }^{42}$ although this was recommended in at least one set of international guidelines on VLU care. ${ }^{2627}$

Limited qualitative studies published in the past indicate that HL may affect VLU patients' self-management capabilities, ${ }^{43-45}$ yet there have been no recent reviews published examining the level of HL of patients with VLUs, and the effect it has on patient's adoption of health behaviours. These studies suggest that inadequate HL reduces the likelihood of engaging in VLU compression, but there is also the possibility that those with lower HL may not increase their physical activity in response to a VLU (despite also being in the recommendations). One educational intervention study $(\mathrm{N}=20)$ indicated that specific HL in VLU is poor, but also demonstrated that there is utility in improving HL in VLU patients. The authors reported that patients felt more confident in VLU management after the educational intervention. ${ }^{46}$ However, this study did not examine physical activity rates, and was hampered by a small sample size. Therefore, most research in this field requires further refinement.

Furthermore, whether or not HL relates to VLU healing outcomes is not currently known. In some studies, examining other illnesses including type 2 diabetes, HL has a marginal effect on health outcomes. For example, two studies on diabetic foot ulcers have found that lower levels of HL were linked to inappropriate self-care and delayed wound healing. ${ }^{47} 48$ Furthermore, the potentially mediating or moderating effects of IT skills ${ }^{49}$ and other psychological concepts, such as self-efficacy ${ }^{50}$ should be considered, as these factors have been suggested to play a role in HL's link to healing outcomes.

Given that HL in VLU patients appears less extensively researched than other factors in VLU healing, we opted to conduct a scoping review ( $\mathrm{ScR})$. The proposed $\mathrm{ScR}$ is 
a flexible method for identifying and discussing information useful for answering our research questions, and allowing a holistic presentation of the available literature on this topic. ${ }^{51}$

\section{Study objective}

The aim of this review is to scope the research examining the level of HL in VLU patients, and how this level may link to self-management behaviours (particularly exercise and compression adherence), and their VLU healing generally. Findings of this ScR will guide the development of clinical practice guidelines on instructing VLU patients according to their level of HL, as well as an assessment instrument for clinicians caring for VLU patients. We anticipate that the findings of this review will aid practitioners and public health officials in developing HL intervention programmes. These programmes may improve VLU care and facilitate evidence-based practise through improved knowledge translation.

\section{Protocol development}

We will conduct the review in accordance with the Preferred Reporting Items for Systematic Reviews and Meta-Analyses extended for scoping review (PRISMA-ScR) outlined in Tricco et a $\tilde{\check{l}}^{2}$ (table 1). Methods for this ScR were developed based on guidelines developed by Levac et $a \bar{l}^{\tilde{3}}$ using the six framework stages as outlined below.

\section{Stage 1: identifying the research question}

Based on the preliminary research, we have developed the following research questions. Our primary research question is:

1. What levels of HL (both general and specific) have been reported in adults with active or past VLUs across outpatient, home care, community and inpatient care? Our secondary research questions are as follows:

1. Is there any relationship between HL and VLU patient adherence to compression and/or exercise?

2. Is there any relationship between HL and VLU patient healing outcomes?

\section{Stage 2: identifying relevant studies}

This search strategy was developed by the research team with guidance from a medical librarian (CF). Eligible studies will be identified from eight databases: The Cochrane Database of Systematic Reviews; The Cochrane Wounds Specialised Register; The Cochrane Central Register of Controlled Trials (CENTRAL) (The Cochrane Library); OvidMEDLINE; OvidMEDLINE (in-process and other non-indexed citations); Ovid EMBASE; EBSCO CINAHL, Embase and Google Scholar. We will also search trial registries, and reference lists of relevant publications for published and ongoing trials. The search will be limited from January 2000 to December 2020. The literature search will be developed using a combination of medical search headings and free text words. Keywords will be identified and selected from similar articles relevant to the population, concept and context of the study. The keywords and search string relevant to Medline via Ovid can be found in online supplemental appendix 1 . The full search will be conducted using Boolean operators and proximity operators, including wildcards, AND, OR, parentheses, quotations and more as per the database used (table 2).

\section{Stage 3: study selection}

We will limit our search to papers published in English from January 2000 to December 2020. Other languages will be excluded because the concept of health, and thus, the concept of HL, varies across languages. ${ }^{545}$ HL studies will be broadly defined to include studies that sought to assess specific (ie, VLU-related) or general health knowledge (ie, where to find general health information). This criterion is deliberately broad, as preliminary searches suggest there is scant literature on this topic. Our study selection will be guided by the following inclusion criteria:

Patients-patients with a current or previous VLU.

Settings-outpatient, community, home care and inpatient care settings where VLU is managed.

Studies-peer-reviewed reviews and studies (qualitative and quantitative) including randomised controlled trials, cohort, case-control, quasi-experimental, cross-sectional, qualitative studies, literature reviews, ScR guidelines, policies and protocols.

Studies that describe knowledge or education or general HL OR an intervention on this with measures at baseline/control group OR a domain relevant to VLU knowledge change will be included. Dependent variables include pre-post knowledge change OR pre-post change in general HL, OR description of HL at that state. For our secondary questions, dependent variables examined will be adherence to exercise recommendations OR adherence to compression in response to a VLU, or the healing rates of those with VLUs.

The following exclusion criteria will apply:

Patients-no diagnosis of VLU. Exclusion of diabetic foot ulcers, pressure ulcers, pressure injuries, vascular insufficiency.

Settings-no non-health care settings (eg, mail out surveys at home).

Studies-narrative reviews, opinions, commentary, conference proceedings. Studies that did not examine knowledge, education or general HL and its relationship with compression adherence will be excluded.

Selection of sources and evidence will take place in four stages.

Step A-initial retrieval, which will be performed by one researcher.

Step B-title screen. Titles that closely meet research aims will be retained. This step will be performed by one researcher.

Step C-abstract screen. Abstracts will be retained if applicable to research aims. This step will be performed by two researchers. 


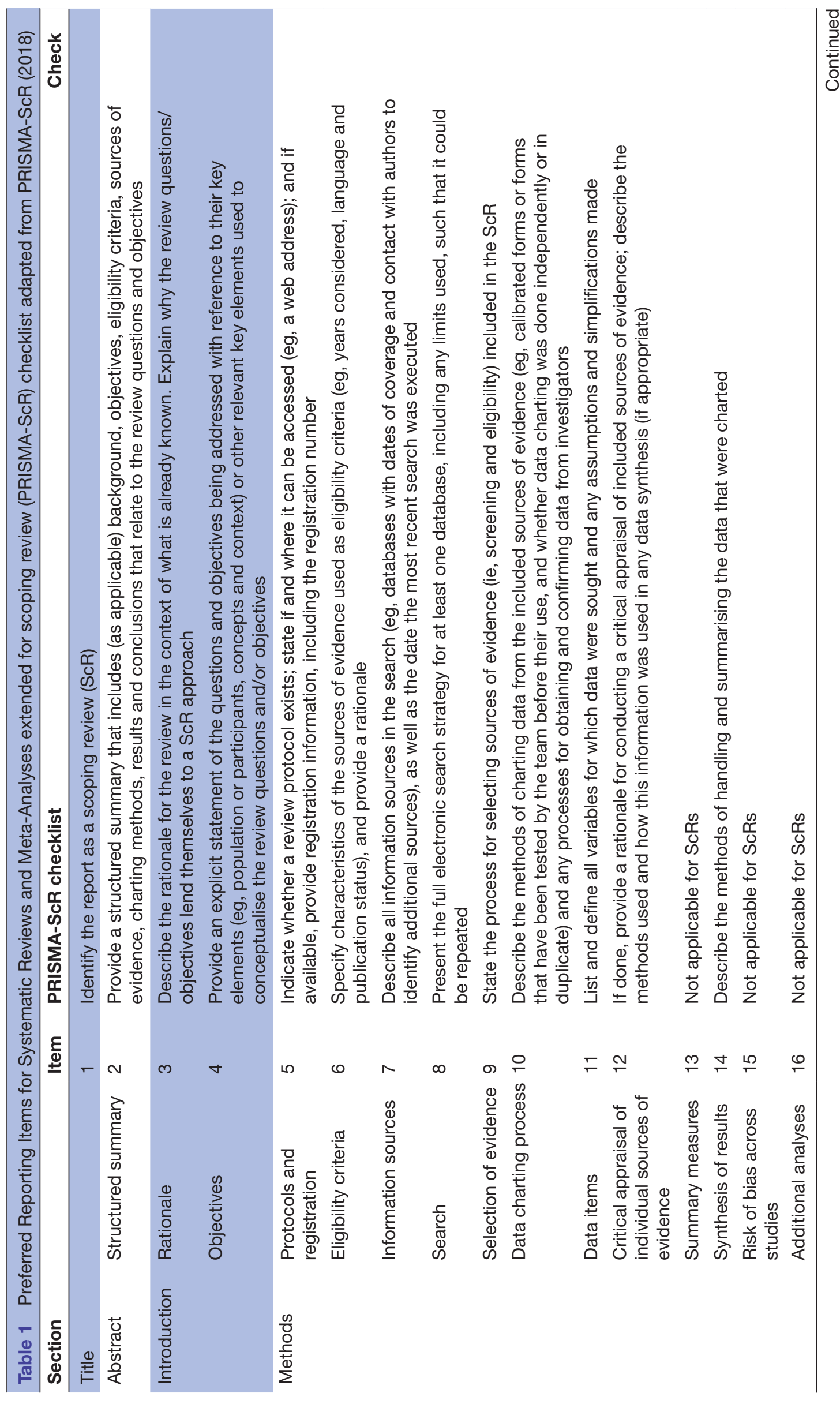




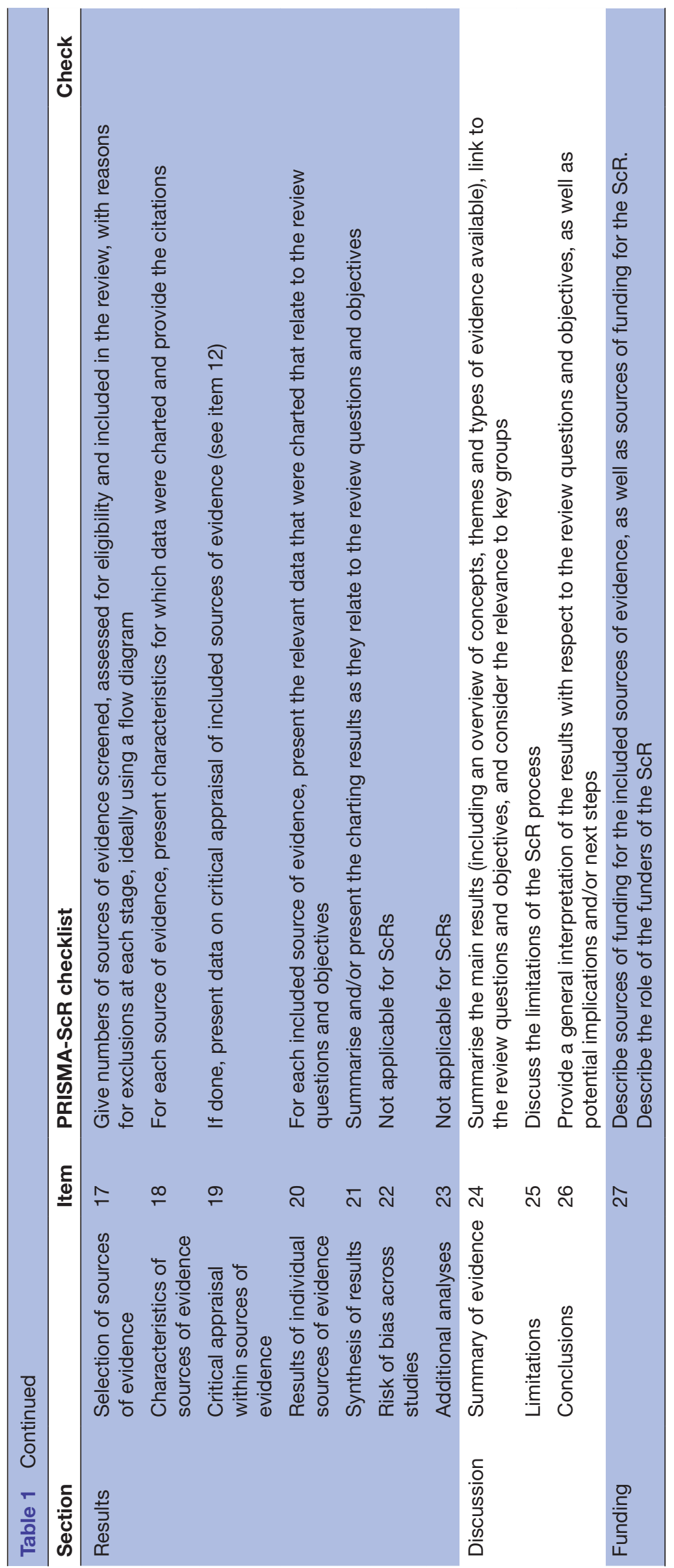

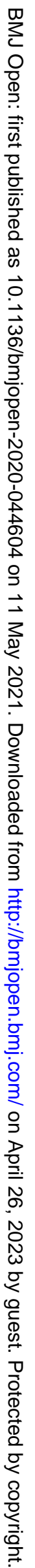




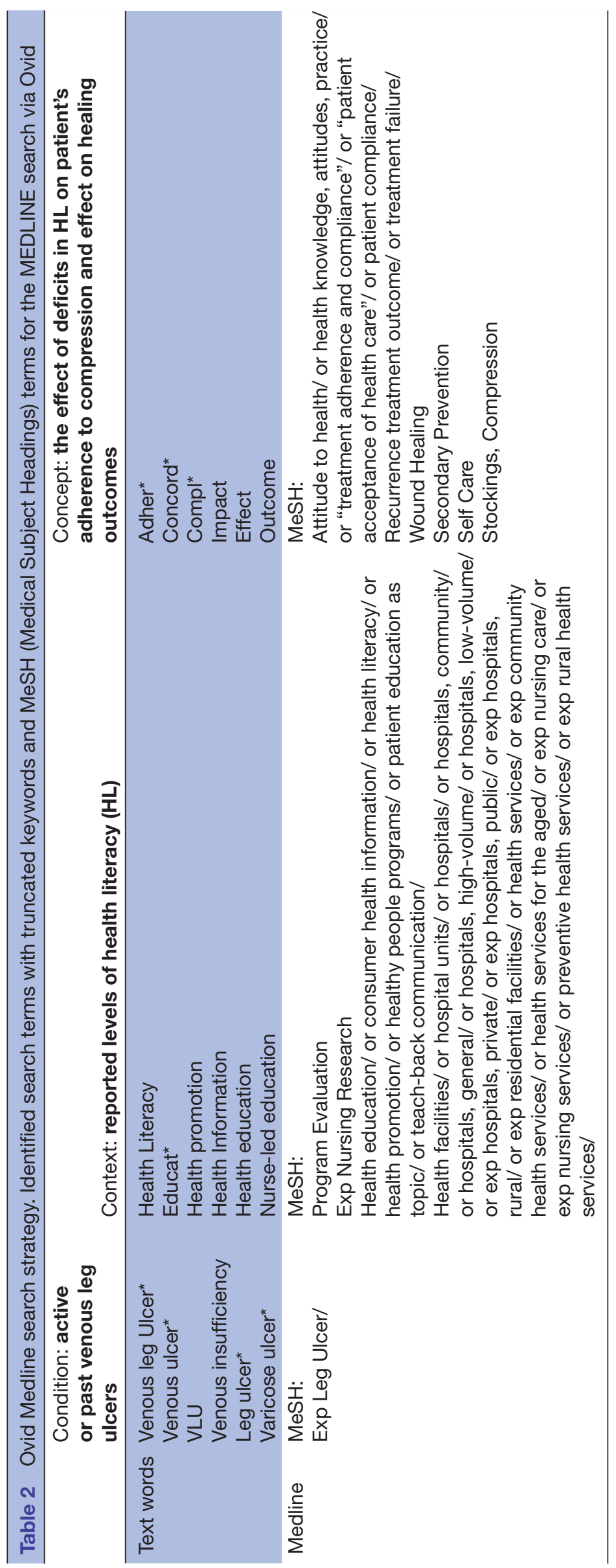




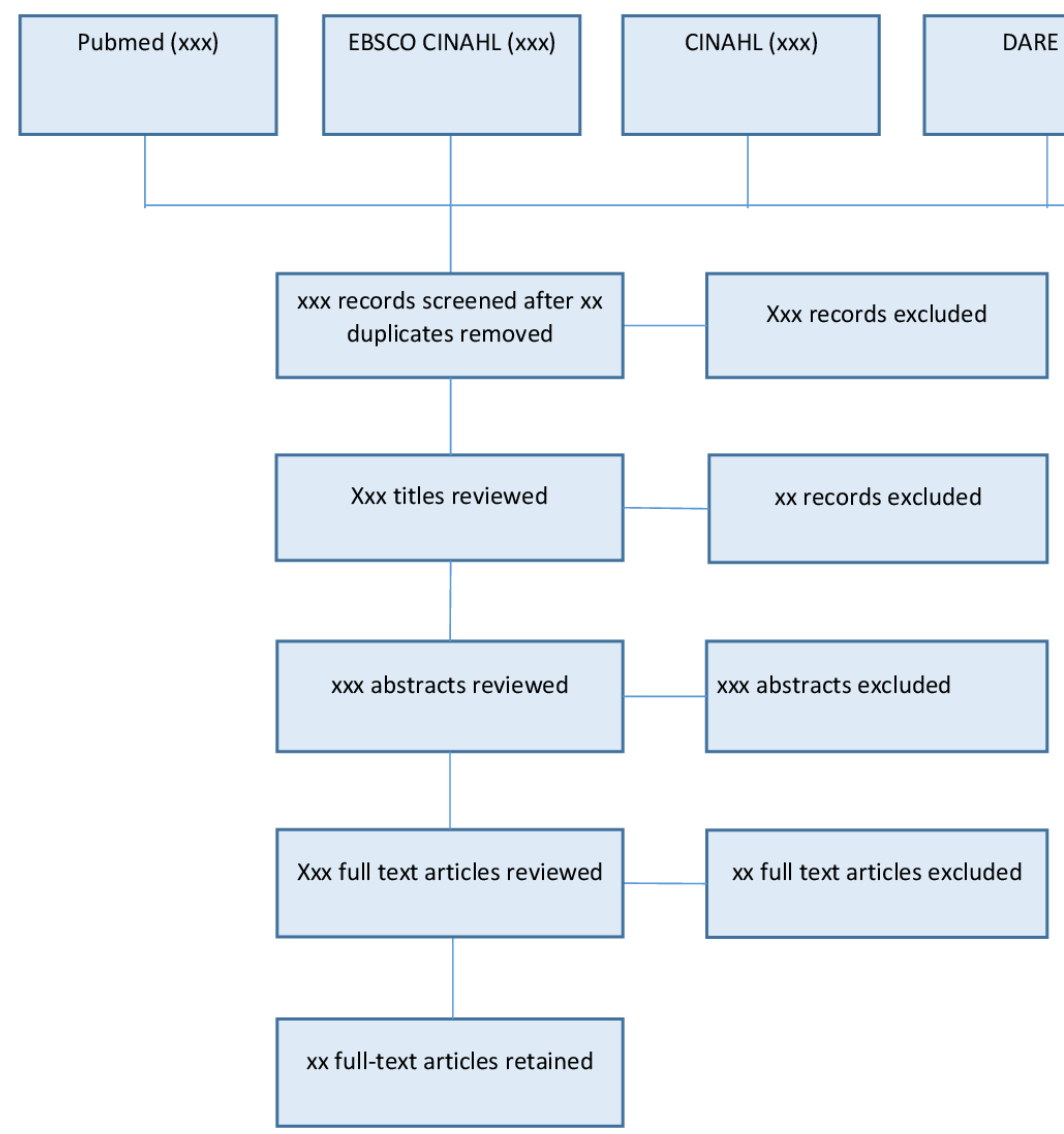

Figure 1 Preferred Reporting Items for Systematic Reviews and Meta-Analyses flowchart.

Step D-full text review. Articles retained if they comply with inclusion and exclusion criteria. This will be performed by all authors of the research team. The numbers of retrieved, assessed, excluded and retained articles will be documented using a PRISMA flowchart (template in figure 1).

Within this step, we will first pilot a sample of 10 studies to ensure that our methods are robust, following to Stage 4 , then re-running a full search again.

\section{Stage 4: charting the data}

An excel spreadsheet and/or covidence will be used to chart the data. This database will record the title and year of the publication, authors, study location, intervention type, study population, aims of the study, overview of methods, outcome measures and results. Duplicates will be removed via Endnote's duplicate detecting function.

\section{Stage 5: collating, summarising and reporting the results}

Full texts that are retained will undergo study quality assessment and critical appraisal in order to determine the applicability of findings to clinical practice. We will use the Critical Skills Appraisal Programme (CASP). ${ }^{56}$ Retained articles will be examined for any qualitative or quantitative descriptions. Findings will be presented in a table that outlines the study type, year the study was undertaken, sample size, study location and patient characteristics.
In order to assess the first research question, we will examine each study against two types of HL: general HL (knowledge or skills) and VLU-specific HL (knowledge or skills). Findings and critical appraisal of the included articles will be provided in a summary of findings and reported in CASP evidence profile table. This table will indicate whether the paper suggested the majority of their sample lacked HL (general or specific). We anticipate that there will be a highly heterogeneous definition of HL in these papers, meaning that authors will be required to use their judgements as to whether or not the paper is relevant, as a degree of simplification will be needed. All entries will be checked by two authors. The lead author will resolve disagreements (if any) independently.

We aim to identify research gaps in the field of VLU treatments by displaying a possible deficit in HL, which translates to lower abilities to adhere to self-management in the form of compression and exercise. The findings are also expected to yield a number of ways HL deficits in VLU patients can be addressed, thereby adding to care and improving standards of care.

\section{Stage 6: consultation-patient and public involvement}

This ScR is the first phase in a multistage research programme aimed at developing a feasibility exercise programme as an adjunct to compression intervention for patients with VLUs. During the consultation phase, we will discuss with people with VLUs and their families 
and caregivers from an established Consumer Wounds Group whether the results of the ScR reflect their needs. The consultation process will take place at the time of a regular consumer group meeting. Data will be gathered using a group interview and Delphi methods. We will map the evidence and identify research gaps and report on compression and exercise treatments by investigating HL, which translates to lower abilities to adhere to self-management in the form of compression and exercise.

\section{Ethics and dissemination}

All articles will be sourced from publicly available platforms. As such, this ScR will not require ethical approval. The findings from the $\mathrm{ScR}$ will be reported in a separate article and submitted to an open-access peer-reviewed journal. The results of this article will provide an outline of the literature, which will be used to inform future research into HL in patients with VLU. To facilitate knowledge translation and our findings, we will liaise with consumer wounds group. The published protocol and final review will be promoted through social media platforms including Twitter and LinkedIn. We will submit the final review at National and International Wound and Health professional Conferences.

\section{Twitter CD Weller @CarolinaDWeller}

Acknowledgements We would like to acknowledge Ms Cassandra Freeman, a librarian from Hargrave-Andrew library, Monash University, for her support with the development a search strategy.

Contributors CDW, SP, GG, JS and LT provided substantial contributions to the conception and design of the work, revisited it critically for important intellectual content, provided final approval of the submitted version and an agreement to be accountable for all aspects of the work in ensuring that questions related to the accuracy or integrity of any part of the work are appropriately investigated and resolved. VT and AB provided substantial contributions to the conception and design of the work, produced the first draft, revisited it critically for important intellectual content, provided final approval of the submitted version and an agreement to be accountable for all aspects of the work in ensuring that questions related to the accuracy or integrity of any part of the work are appropriately investigated and resolved. CR provided substantial contributions to the conception and design of the work, produced the first draft, with the support of a librarian has developed a search strategy, provided final approval of the submitted version and an agreement to be accountable for all aspects of the work in ensuring that questions related to the accuracy or integrity of any part of the work are appropriately investigated and resolved.

Funding CDW was supported by the grant from the Australian National Health and Medical Research Council (APP1069329). Other co-authors received no specific grant from any funding agency in the public, commercial or not-for-profit sectors.

Guarantor of the review Professor Carolina Weller.

Competing interests None declared.

Patient consent for publication Not required.

Provenance and peer review Not commissioned; externally peer reviewed.

Supplemental material This content has been supplied by the author(s). It has not been vetted by BMJ Publishing Group Limited (BMJ) and may not have been peer-reviewed. Any opinions or recommendations discussed are solely those of the author(s) and are not endorsed by BMJ. BMJ disclaims all liability and responsibility arising from any reliance placed on the content. Where the content includes any translated material, BMJ does not warrant the accuracy and reliability of the translations (including but not limited to local regulations, clinical guidelines, terminology, drug names and drug dosages), and is not responsible for any error and/or omissions arising from translation and adaptation or otherwise.
Open access This is an open access article distributed in accordance with the Creative Commons Attribution Non Commercial (CC BY-NC 4.0) license, which permits others to distribute, remix, adapt, build upon this work non-commercially, and license their derivative works on different terms, provided the original work is properly cited, appropriate credit is given, any changes made indicated, and the use is non-commercial. See: http://creativecommons.org/licenses/by-nc/4.0/.

\section{ORCID iDs}

CD Weller http://orcid.org/0000-0002-8016-1060

Victoria Team http://orcid.org/0000-0001-6615-6874

Sebastian Probst http://orcid.org/0000-0001-9603-1570

Georgina Gethin http://orcid.org/0000-0001-5859-8357

Jane Sixsmith http://orcid.org/0000-0002-3212-8186

Ayoub Bouguettaya http://orcid.org/0000-0002-5843-3533

\section{REFERENCES}

1 Australian Wound Management Association Inc, New Zealand Wound Care Society Inc. Australian and New Zealand clinical practice guideline for prevention and management of venous leg ulcers. Barton, ACT: Cambridge Publishing, 2011.

2 Domingues EAR, Kaizer UAO, Lima MHM. Effectiveness of the strategies of an orientation programme for the lifestyle and woundhealing process in patients with venous ulcer: a randomised controlled trial. Int Wound J 2018;15:798-806.

3 Graves N, Zheng $\mathrm{H}$. The prevalence and incidence of chronic wounds: a literature review. Wound Practice Res 2014;22:4-12.

4 Xie T, Ye J, Rerkasem K, et al. The venous ulcer continues to be a clinical challenge: an update. Burns Trauma 2018;6:18.

5 Meulendijks AM, Franssen WMA, Schoonhoven L, et al. A scoping review on chronic venous disease and the development of a venous leg ulcer: the role of obesity and mobility. J Tissue Viability 2020;29:190-6.

6 Weller CD, Team V, Ivory JD, et al. ABPI reporting and compression recommendations in global clinical practice guidelines on venous leg ulcer management: a scoping review. Int Wound J 2019;16:406-19.

7 Pérez MB, López-Casanova P, Lavín RS. Recent Reports from University of Alicante Highlight Findings in Leg Ulcers [Epidemiology of venous leg ulcers in primary health care: Incidence and prevalence in a health centre-A time series study (2010-2014)] Citation metadata. Int Wound J 2018;16:256-65.

8 Jockenhöfer F, Gollnick H, Herberger K, et al. Aetiology, comorbidities and cofactors of chronic leg ulcers: retrospective evaluation of 1000 patients from 10 specialised dermatological wound care centers in Germany. Int Wound J 2016;13:821-8.

9 Thistlethwaite KR, Finlayson KJ, Cooper PD, et al. The effectiveness of hyperbaric oxygen therapy for healing chronic venous leg ulcers: a randomized, double-blind, placebo-controlled trial. Wound Repair Regen 2018;26:324-31.

10 Finlayson KJ, Parker CN, Miller C, et al. Predicting the likelihood of venous leg ulcer recurrence: the diagnostic accuracy of a newly developed risk assessment tool. Int Wound J 2018;15:686-94.

11 Santler B, Goerge T. Chronic venous insufficiency - a review of pathophysiology, diagnosis, and treatment. J Dtsch Dermatol Ges 2017:15:538-56.

12 Weller C, Ademi Z, Makarounas-Kirchmann K. Economic evaluation of compression therapy in venous leg ulcer randomised controlled trials: a systematic review. Wound Practice Res 2012;20:21.

13 Cheng Q, Gibb M, Graves N, et al. Cost-Effectiveness analysis of guideline-based optimal care for venous leg ulcers in Australia. BMC Health Serv Res 2018;18:421.

14 Nussbaum SR, Carter MJ, Fife CE, et al. An economic evaluation of the impact, cost, and Medicare policy implications of chronic nonhealing wounds. Value Health 2018;21:27-32.

15 Ruseckaite R, Richards C, Rutherford C, et al. A conceptual framework of patient-reported outcomes in people with venous leg ulcers. Wound Repair Regen 2020;28:355-63.

16 Cheng Q, Kularatna S, Lee XJ, et al. Comparison of EQ-5D-5L and SPVU-5D for measuring quality of life in patients with venous leg ulcers in an Australian setting. Qual Life Res 2019;28:1903-11.

17 Barnsbee L, Cheng Q, Tulleners R, et al. Measuring costs and quality of life for venous leg ulcers. Int Wound J 2019;16:112-21.

18 Pacella R, Tulleners R, Cheng Q. Solutions to the chronic wounds problem in Australia: a call to action. Wound Practice Res 2018;26:84-98.

19 Australian Wound Management Association. KPMG Health Economics report: An economic evaluation of compression therapy 
for venous leg ulcers. Canberra: Australian Wound Management Association, 2013.

20 Andriessen A, Apelqvist J, Mosti G, et al. Compression therapy for venous leg ulcers: risk factors for adverse events and complications, contraindications - a review of present guidelines. J Eur Acad Dermatol Venereol 2017;31:1562-8.

21 O'Meara S, Cullum N, Nelson EA, et al. Compression for venous leg ulcers. Cochrane Database Syst Rev 2012;11:CD000265.

22 Team V, Chandler PG, Weller CD. Adjuvant therapies in venous leg ulcer management: a scoping review. Wound Repair Regen 2019;27:562-90.

23 Klonizakis M, Tew GA, Gumber A, et al. Supervised exercise training as an adjunct therapy for venous leg ulcers: a randomized controlled feasibility trial. Br J Dermatol 2018;178:1072-82.

24 Jull A, Slark J, Parsons J. Prescribed exercise with compression vs compression alone in treating patients with venous leg ulcers: a systematic review and meta-analysis. JAMA Dermatol 2018:154:1304-11.

25 Orr L, Klement KA, McCrossin L, et al. A systematic review and meta-analysis of exercise intervention for the treatment of calf muscle pump impairment in individuals with chronic venous insufficiency. Ostomy Wound Manage 2017;63:30-43.

26 Franks PJ, Barker J, Collier M, et al. Management of patients with venous leg ulcers: challenges and current best practice. J Wound Care 2016;25 Suppl 6:S1-67.

27 Gethin G, Probst S, Stryja J, et al. Evidence for person-centred care in chronic wound care: a systematic review and recommendations for practice. J Wound Care 2020;29:S1-22.

28 Boxall S, Carville K, Leslie G, et al. Compression bandaging: identification of factors contributing to non-concordance. Wound Practice Res 2019;27:6-20.

29 Yamashita T, Kart CS. Is diabetes-specific health literacy associated with diabetes-related outcomes in older adults? J Diabetes 2011;3:138-46.

30 Muldoon J. Compression hosiery for venous conditions: a literature review. J Community Nurs 2019;33:29-34.

31 Sørensen K, Pelikan JM, Röthlin F, et al. Health literacy in Europe: comparative results of the European health literacy survey (HLS-EU). Eur J Public Health 2015;25:1053-8.

32 Shi C, Dumville JC, Cullum N, et al. Compression bandages or stockings versus no compression for treating venous leg ulcers. Cochrane Database Syst Rev 2019;18.

33 Australian Institute of Health and Welfare. Australia's health 2018. Australia's health series no. 16 AUS 221. Canberra: AlHW, 2018.

34 Baker DW, Wolf MS, Feinglass J, et al. Health literacy, cognitive abilities, and mortality among elderly persons. J Gen Intern Med 2008;23:723-6.

35 Yamashita T, Bardo AR, Liu D, et al. Literacy, numeracy, and health information seeking among middle-aged and older adults in the United States. J Aging Health 2020;32:33-41.

36 Turner AM, Osterhage KP, Taylor JO, et al, eds. A closer look at health information seeking by older adults and involved family and friends: design considerations for health information technologies. AMIA annual symposium proceedings. Maryland: American Medical Informatics Association, 2018.
37 Chaudhuri S, Le T, White C, et al. Examining health informationseeking behaviors of older adults. Comput Informatics Nurs 2013;31:547-53.

38 Chase SK, Melloni M, Savage A. A forever healing: the lived experience of venous ulcer disease. J Vasc Nurs 1997;15:73-8.

39 Shanley E, Moore Z, Patton D, et al. Patient education for preventing recurrence of venous leg ulcers: a systematic review. J Wound Care 2020;29:79-91.

40 Berkman ND, Sheridan SL, Donahue KE, et al. Low health literacy and health outcomes: an updated systematic review. Ann Intern Med 2011;155:97-107.

41 MacLeod S, Musich S, Gulyas S, et al. The impact of inadequate health literacy on patient satisfaction, healthcare utilization, and expenditures among older adults. Geriatr Nurs 2017;38:334-41.

42 Graham S, Brookey J. Do patients understand? Perm J 2008:12:67.

43 Walshe C. Living with a venous leg ulcer: a descriptive study of patients' experiences. J Adv Nurs 1995;22:1092-100.

44 Chase SK, Whittemore R, Crosby N, et al. Living with chronic venous leg ulcers: a descriptive study of knowledge and functional health status. J Commun Health Nurs 2000;17:1-13.

45 Probst S, Séchaud L, Bobbink P, et al. The lived experience of recurrence prevention in patients with venous leg ulcers: an interpretative phenomenological study. J Tissue Viability 2020;29:176-9.

46 CLARKE C, WHITMORE L, WEBB A. Patient education pictorial boards: improving patients' understanding of venous leg ulcer and compression therapy. Wounds UK 2020;16:54-60.

47 Margolis DJ, Hampton M, Hoffstad O, et al. Health literacy and diabetic foot ulcer healing. Wound Repair Regen 2015;23:299-301.

48 Lael-Monfared E, Tehrani H, Moghaddam ZE, et al. Health literacy, knowledge and self-care behaviors to take care of diabetic foot in low-income individuals: application of extended parallel process model. Diabetes Metab Syndr 2019;13:1535-41.

49 Boren SA. A review of health literacy and diabetes: opportunities for technology. J Diabetes Sci Technol 2009;3:202-9.

50 Xu XY, Leung AYM, Chau PH. Health literacy, self-efficacy, and associated factors among patients with diabetes. Health Lit Res Pract 2018;2:e67-77.

51 Pham MT, Rajić A, Greig JD, et al. A scoping review of scoping reviews: advancing the approach and enhancing the consistency. Res Synth Methods 2014;5:371-85.

52 Tricco AC, Lillie E, Zarin W, et al. PRISMA extension for scoping reviews (PRISMA-ScR): checklist and explanation. Ann Intern Med 2018;169:467-73.

53 Levac D, Colquhoun H, O'Brien KK. Scoping studies: advancing the methodology. Implement Sci 2010;5:69.

54 Choi TST, Walker KZ, Ralston RA, et al. Diabetes education needs of Chinese Australians: a qualitative study. Health Educ $J$ 2015;74:197-208.

55 Walker C, Weeks A, McAvoy B, et al. Exploring the role of selfmanagement programmes in caring for people from culturally and linguistically diverse backgrounds in Melbourne, Australia. Health Expect 2005;8:315-23.

56 Singh J. Critical appraisal skills programme. J Pharmacol Pharmacother 2013;4:76. 